\title{
Treating refractory dermatomyositis or polymyositis with adrenocorticotropic hormone gel: a retrospective case series
}

This article was published in the following Dove Press journal:

Drug Design, Development and Therapy

9 June 2012

Number of times this article has been viewed

\author{
Todd Levine \\ Phoenix Neurological Associates, \\ Phoenix, AZ, USA
}

Background: Effective and tolerable treatment options for patients with dermatomyositis and polymyositis are limited. This retrospective case review describes treatment with adrenocorticotropic hormone (ACTH) gel in five patients who experienced a disease exacerbation and either failed or were unable to tolerate the side effects of previous therapy with steroids, intravenous immunoglobulins, and steroid-sparing drugs.

Methods: Patients received ACTH gel subcutaneous injections of $80 \mathrm{U}(1 \mathrm{~mL})$ twice weekly (four patients) or once weekly (one patient) over the course of 12 weeks for short-term treatment of symptom exacerbations. Manual muscle testing using the Medical Research Council scale was assessed at baseline and at 3 months.

Results: Improvement was seen in all patients, including improved muscle strength, decreased pain, and resolution of skin involvement. All patients tolerated the treatment well, and no significant side effects occurred.

Conclusion: The treatment of dermatomyositis and polymyositis is an approved use for ACTH gel, and these anecdotal reports would suggest consideration of ACTH gel as a therapeutic option. Further investigation is warranted.

Keywords: adrenocorticotropic hormone gel, dermatomyositis, polymyositis, steroids, intravenous immunoglobulins

\section{Introduction}

Dermatomyositis and polymyositis are systemic inflammatory disorders characterized by symmetric proximal muscle weakness and commonly involve other organ systems, such as the skin (in dermatomyositis) and lung. ${ }^{1}$ Treatment decisions are typically empirically based due to few controlled trials and a lack of targeted immunosuppression. Expert consensus supports high-dose oral prednisone as first-line therapy; however, as many as $30 \%-40 \%$ of patients may fail to respond, and up to $40 \%$ or more experience major adverse events with long-term steroid use. ${ }^{2}$ Steroid-sparing or alternative immunosuppressive therapies, including methotrexate, azathioprine, cyclosporine, and mycophenolate mofetil, have a long onset of action and risks, including toxicity to the kidneys, liver, and bone marrow. ${ }^{1}$ Intravenous immunoglobulin is considered a secondline therapy for dermatomyositis, but not for polymyositis. ${ }^{3}$ However, intravenous immunoglobulin does not have a US Food and Drug Administration (FDA) indication for myositis, and is very expensive with a risk of acute renal failure. ${ }^{1,4}$ Rituximab had shown some promise in anecdotal case series, but a large, international, multisite, randomized, controlled clinical trial (Rituximab in Myositis) showed no separation from placebo. ${ }^{5,6}$ Clearly, additional effective and tolerable treatment options are needed. 
Adrenocorticotropic hormone (ACTH) gel (HP Acthar ${ }^{\circledR}$ Gel, repository corticotropin injection, Questcor Pharmaceuticals, Inc., Union City, CA) is a long-acting formulation of the full sequence $\mathrm{ACTH}_{(1-39)}$ that includes other pro-opiomelanocortin peptides. Dermatomyositis and polymyositis are approved uses of ACTH gel that were granted when the product was first approved in $1952 .{ }^{7}$ In 2010, the FDA reviewed and modernized the entire ACTH gel label alongside granting a new indication for infantile spasms, and the dermatomyositis and polymyositis indications were retained as approved uses. Despite FDA approval, clinical data are limited and many physicians treating these disorders are unaware of ACTH gel as a treatment option approved by the FDA. Therefore, ACTH gel represents a novel, approved therapeutic option for these disease states.

Emerging evidence related to the melanocortin system suggests that ACTH may have mechanisms of action in addition to steroidogenesis, resulting in anti-inflammatory and immunomodulatory effects. Five melanocortin receptors are known to be expressed on a variety of cells, including immune cells, glial cells, and podocytes. Through these receptors, melanocortins can induce a broad range of immune-modulating effects. ${ }^{8}$ Similarly, mechanisms of action now being considered for ACTH gel hypothesize effects beyond steroidogenesis. ACTH gel has been shown to be effective in patients refractory to steroids and other therapies in infantile spasms, nephrotic syndrome, and acute exacerbations of multiple sclerosis, with suggested immunemodulating mechanisms of action. ${ }^{-11}$

The retrospective case series presented here provides clinical observations relevant to treating patients with biopsy-confirmed, highly refractory dermatomyositis and polymyositis using the ACTH gel formulation described above. Five patients (three with dermatomyositis, two with polymyositis) with disease exacerbation who had failed or were unable to tolerate the side effects of previous therapy are described.

\section{Materials and methods}

All patients in this retrospective case series review were female, aged 25-68 years, with diagnoses confirmed on muscle biopsy (see Table 1). ACTH gel was approved and paid for by each patient's health insurance. All patients received $80 \mathrm{U}(1 \mathrm{~mL})$ of ACTH gel via subcutaneous injection. Four patients received ACTH gel twice weekly for 12 weeks and one patient received ACTH gel once weekly. The dosing regimen used with these patients was based on previous studies of ACTH gel in patients with exacerbations of muscle sclerosis and nephrotic syndrome. ${ }^{10,11}$ Manual muscle testing, recommended in the assessment of treatment outcomes in patients with dermatomyositis and polymyositis, was performed using the Medical Research Council scale at baseline and at 3 months. ${ }^{12-14} \mathrm{~A}$ Medical Research Council manual muscle testing scale score of 2 reflects an inability to produce active movement against gravity, a score of 3 reflects active movement against gravity but not resistance, a score of 4 reflects active movement against gravity and resistance, and a score of 5 reflects normal power. Muscles tested included deltoids, biceps, triceps, wrist extensors, first dorsal interossei, grip strength, iliopsoas, quadriceps, hamstring, and tibialis anterior. Muscle groups that improved are described below. No muscles weakened from baseline testing during ACTH gel treatment, and muscles that remained the same are not discussed. Previous therapies and concomitant medications are summarized in Table 1. All patients had received stable dosing of concomitant therapy for a minimum of 60 days, and the treatment had failed to treat the disease exacerbation adequately before receiving ACTH gel. Patients were kept on existing treatments during $\mathrm{ACTH}$ gel treatment so that following short-term treatment with ACTH gel to quiet the disease exacerbation, the patients could continue their previous treatment regimen for longer-term maintenance therapy. Patients were examined for significant side effects in response to ACTH gel, including hyperglycemia, diabetic ketoacidosis, and hyperosmolar states. Glycosylated hemoglobin $\left(\mathrm{HbA}_{1 \mathrm{c}}\right)$ was assessed monthly. This retrospective review of the treatment received by patients during clinical care of dermatomyositis and polymyositis received institutional review board exemption from Western Institutional Review Board.

\section{Case reports Patient I}

A 45-year-old woman diagnosed with dermatomyositis presented with a diffuse erythematous rash across her chest and finger extensors, clear periungual telangiectasias, and mechanic's hands, with progressive arm and leg muscle weakness. She had an elevated creatine phosphokinase of 4500 , and muscle biopsy showed perifascicular atrophy and inflammation. Autoantibody testing showed no myositisspecific autoantibodies.

The patient's treatment history since 2004 included intravenous immunoglobulin, azathioprine, rituximab, cyclosporine, and methotrexate. The patient initially responded well to treatment with prednisone $\leq 40 \mathrm{mg}$ /day (the patient would not agree to take $>40 \mathrm{mg} /$ day of prednisone 


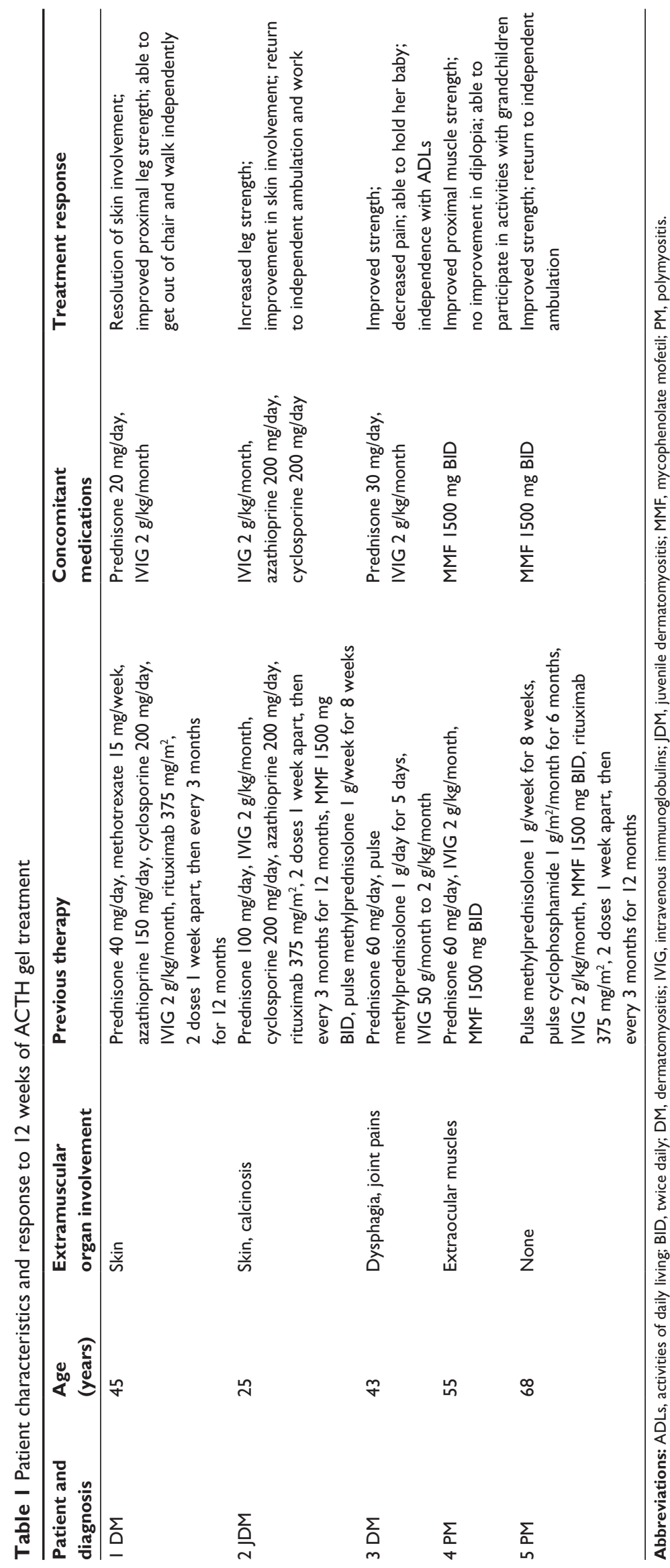


due to side effects of weight gain, mood changes, and severe gastric symptoms), intravenous immunoglobulin $2 \mathrm{~g} / \mathrm{kg}$ per month (she experienced moderate side effects to intravenous immunoglobulin, including headache, chills, and nausea), and azathioprine $150 \mathrm{mg}$ /day. In 2007, she experienced a disease exacerbation, and rituximab $375 \mathrm{mg} / \mathrm{m}^{2}$ for two doses and then a single dose every 3 months for 12 months was added to ongoing prednisone, azathioprine, and intravenous immunoglobulin. The patient showed good improvement in her rash and muscle strength. Her disease became more active in 2009, including muscle weakness, severe rash, and significant nail bed changes, and she had an inadequate response to treatment with prednisone, intravenous immunoglobulin, cyclosporine $200 \mathrm{mg} /$ day, and methotrexate $15 \mathrm{mg} /$ week.

The patient received treatment with ACTH gel concomitantly with prednisone $20 \mathrm{mg}$ /day and intravenous immunoglobulin $2 \mathrm{~g} / \mathrm{kg}$ per month. Her creatine phosphokinase levels prior to starting ACTH gel therapy were normal. By 8 weeks, her skin rash had improved markedly. Proximal leg strength increased from $3 / 5$ to $4+/ 5$ in her iliopsoas and quadriceps. She was able to get out of a chair and walk independently. The patient tolerated the treatment well without any significant side effects. $\mathrm{HbA}_{1 \mathrm{c}}$ remained normal. Since completing ACTH gel treatment, she has been managed adequately with intravenous immunoglobulin $2 \mathrm{~g} / \mathrm{kg}$ per month, prednisone $20 \mathrm{mg} /$ day, and methotrexate $15 \mathrm{mg} /$ week.

\section{Patient 2}

A 25-year-old woman diagnosed with juvenile dermatomyositis presented at the age of 11 years with muscle weakness, classic skin changes, and calcinosis. She had an elevated creatine phosphokinase of 56,000 and a muscle biopsy showing perifascicular atrophy and inflammation. Autoantibody testing showed no myositis-specific autoantibodies. At the time of transition to adult care in 2004, she was very weak and required the use of a walker.

Her treatment history as a pediatric patient included prednisone up to $100 \mathrm{mg} /$ day, resulting in significant cushingoid side effects, mycophenolate mofetil $3000 \mathrm{mg} /$ day, which resulted in palpitations, azathioprine $200 \mathrm{mg} /$ day, intravenous immunoglobulin $2 \mathrm{~g} / \mathrm{kg}$ per month, cyclosporine $200 \mathrm{mg} /$ day, and methotrexate $20 \mathrm{mg} /$ week. Since transitioning to adult care, she had received rituximab, cyclosporine, intravenous immunoglobulin, azathioprine, and pulse methylprednisolone. The patient showed a good response to rituximab (375 $\mathrm{mg} / \mathrm{m}^{2}$ for two doses and then a single dose every 3 months for 12 months), and was maintained on intravenous immunoglobulin, cyclosporine, and azathioprine until a disease exacerbation in 2007. The patient did not show improvement on rechallenge with rituximab over the next 6 months; her strength worsened, and she again required a walker for ambulation. She did not show improvement in response to pulse methylprednisolone $1 \mathrm{~g}$ /week for 8 weeks.

The patient received $\mathrm{ACTH}$ gel concomitant with ongoing intravenous immunoglobulin $2 \mathrm{~g} / \mathrm{kg}$ per month, azathioprine $200 \mathrm{mg} /$ day, and cyclosporine $200 \mathrm{mg}$ /day. Her creatine phosphokinase and aldolase levels prior to initiation of ACTH gel were in the low-normal range, reflecting significant previous muscle atrophy. The patient noted improved strength and independent ambulation within 8 weeks of treatment. Her muscle enzymes decreased from 800 to 100 . The patient's iliopsoas strength improved from $3 / 5$ to $4 / 5$ and her quadriceps strength increased from $3-/ 5$ to $4+/ 5$. She also reported improvement in her rash and nail bed changes. Within 6 months of completing ACTH gel, and while on intravenous immunoglobulin and azathioprine, weakness in her proximal leg muscles again declined to $3+/ 5$. She was started on tacrolimus for 6 months with no benefit. The patient was restarted on ACTH gel, $80 \mathrm{U}$ once a week with partial improvement in strength. Treatment with ACTH gel was increased to $80 \mathrm{U}$ twice weekly, and the patient has shown a good response. Her leg muscle strength improved to $4+/ 5$. She has continued on ACTH gel for 7 months, in combination with intravenous immunoglobulin and azathioprine. Her strength is $5 / 5$ in all muscle groups except her iliopsoas, where it is $4 / 5$. The patient has been able to return to work and is ambulating independently. The patient tolerated ACTH gel treatment well without any significant side effects. Her $\mathrm{HbA}_{1 \mathrm{c}}$ and fasting glucose levels have remained normal, and there has been no change in her bone density as measured by densitometry.

\section{Patient 3}

A 43-year-old woman diagnosed with dermatomyositis presented with diffuse erythematous rash, profound diffuse muscle weakness, and joint pains. The patient had an elevated creatine phosphokinase of 2500, and muscle biopsy showed perifascicular atrophy and inflammation. Autoantibody testing showed no myositis-specific autoantibodies.

Treatment history since 2009 included prednisone up to $60 \mathrm{mg} /$ day, weekly pulse methylprednisolone $1 \mathrm{~g} /$ day, and intravenous immunoglobulin as high as $2 \mathrm{~g} / \mathrm{kg}$ per month. Rituximab was denied by the patient's insurance company. The patient became pregnant in 2010, and during pregnancy and subsequent childbirth, she experienced a marked decrease 
in muscle strength and difficulty walking. Lower extremity strength responded well to pulse methylprednisolone $1 \mathrm{~g} /$ day for 5 days, prednisone $60 \mathrm{mg} /$ day, and monthly intravenous immunoglobulin $50 \mathrm{~g}$ increased to $2 \mathrm{~g} / \mathrm{kg}$. However, upper extremity muscle weakness continued, and she was not able to hold her baby. The patient disliked high-dose prednisone due to side effects of significant weight gain and elevated glucose. She also experienced severe side effects to highdose intravenous immunoglobulin, including rash, headache, fevers, and malaise.

Treatment with ACTH gel was initiated concomitant with prednisone $30 \mathrm{mg} /$ day and intravenous immunoglobulin $2 \mathrm{~g} / \mathrm{kg}$ per month. Her creatine phosphokinase levels prior to starting therapy were mildly elevated at 647 . The patient's diffuse strength increased after 12 weeks and was accompanied by a drop in her creatine phosphokinase to 154 . She was able to hold her baby and she became independent with her activities of daily living. Most notably, deltoid strength increased from $2 / 5$ to $4 / 5$ and triceps strength from $3 / 5$ to $4 / 5$. Her myalgias also improved markedly. The patient tolerated the treatment well without any significant side effects or change in $\mathrm{HbA}_{1 \mathrm{c}}$. Since completing ACTH gel, she has been managed adequately with prednisone $35 \mathrm{mg}$ /day and intravenous immunoglobulin $50 \mathrm{~g}$ per month.

\section{Patient 4}

A 55-year-old woman diagnosed with polymyositis presented with marked limitation of her extraocular muscles bilaterally and mild proximal muscle weakness in her arms. She had a mildly elevated creatine phosphokinase of 650 , and a muscle biopsy of the right deltoid showed inflammatory changes consistent with polymyositis. An orbital magnetic resonance imaging scan showed inflammation of the extraocular muscles. Testing for acetylcholine receptor antibodies and antithyroid antibodies, an electromyogram with repetitive nerve stimulation and single-fiber testing, and thyroid profile were all normal. Genetic testing for oculopharyngeal muscular dystrophy was normal. Autoantibody testing showed a positive rheumatoid factor, a positive antinuclear antibody 1:1260, and no myositis-specific autoantibodies.

Treatment history since 2007 included prednisone, mycophenolate mofetil, and intravenous immunoglobulin. The patient was initially treated with prednisone $60 \mathrm{mg} /$ day. Her eye movements and double vision responded very well, and prednisone was tapered to $20 \mathrm{mg} /$ day. The patient experienced a disease exacerbation in 2009 with increased arm and leg weakness that prevented her from engaging in activities with her grandchildren. Prednisone was increased to $60 \mathrm{mg} /$ day and resulted in improved muscle strength; however, the patient strongly disliked prednisone due to mood-related side effects. Mycophenolate mofetil $1500 \mathrm{mg}$ twice daily was added to treatment and prednisone was tapered and withdrawn. The patient's strength and eye movements again worsened, and intravenous immunoglobulin $2 \mathrm{~g} / \mathrm{kg}$ per month for 6 months showed no benefit.

The patient began treatment with ACTH gel concomitant with ongoing mycophenolate mofetil $1500 \mathrm{mg}$ twice daily. She had normal creatine phosphokinase and aldolase levels prior to starting therapy with ACTH gel. Proximal muscle strength increased after 12 weeks; most notably, the patient's deltoids and triceps improved from $3 / 5$ to $4+/ 5$. No improvement occurred in her extraocular muscles, but the patient reported that she was again able to participate in activities with her grandchildren. The patient tolerated the treatment well, without any significant side effects or increase in $\mathrm{HbA}_{1 \mathrm{c}}$. Since completing ACTH gel, she has been managed adequately with intravenous immunoglobulin $2 \mathrm{~g} / \mathrm{kg}$ per month and mycophenolate mofetil $1500 \mathrm{mg}$ twice daily.

\section{Patient 5}

A 68-year-old woman diagnosed with polymyositis presented with muscle weakness, difficulty getting out of a chair and climbing stairs, and weak grip strength that was reduced to $40 \mathrm{lb}$. Myositis-specific autoantibody tests were normal. Muscle biopsy of the quadriceps was negative for inclusion body myositis and was consistent with polymyositis.

The patient's treatment history since 2003 included pulse methylprednisolone, pulse cyclophosphamide, intravenous immunoglobulin, mycophenolate mofetil, and rituximab. The patient responded well to initial combination treatment with pulse methylprednisolone $1 \mathrm{~g} /$ week for 8 weeks and pulse cyclophosphamide $1 \mathrm{~g} / \mathrm{m}^{2}$ per month for 6 months. Treatment with pulse methylprednisolone was stopped due to recurrent infections, including hospitalization for severe pneumonia, and intravenous immunoglobulin $2 \mathrm{~g} / \mathrm{kg}$ per month was started. The patient responded well to combination treatment with monthly pulse cyclophosphamide and intravenous immunoglobulin. The patient was weaned off intravenous immunoglobulin, and mycophenolate mofetil $1500 \mathrm{mg}$ twice daily was added. In 2008, the patient experienced a disease exacerbation, and rituximab $375 \mathrm{mg} / \mathrm{m}^{2}$ for two doses and then a single dose every 3 months for 12 months was added to ongoing mycophenolate mofetil. In 2011, the patient again experienced disease exacerbation that improved with pulse methylprednisolone and mycophenolate mofetil. Medicare then refused payment for continued pulse 
methylprednisolone, and the patient had 3 months without steroid therapy. During this time, her energy and strength worsened and she returned to occasional use of a walker.

The patient began treatment with ACTH gel $80 \mathrm{U}$ once weekly, concomitant with ongoing mycophenolate mofetil $1500 \mathrm{mg}$ twice daily. Her creatine phosphokinase levels prior to starting therapy with ACTH gel were mildly elevated at 632. After 12 weeks of treatment with ACTH gel, her creatine phosphokinase decreased to 388 . She tolerated the treatment well, without an increase in $\mathrm{HbA}_{1 \mathrm{c}}$ or any weight gain, sleep disturbance, or gastrointestinal symptoms, which had been significant side effects while on pulse methylprednisolone. The patient noted significant improvement in her strength and no longer required the use of a walker. Her muscle strength improved from $4 / 5$ to $5 / 5$ in her quadriceps and to $4+/ 5$ in her iliopsoas. The patient will continue ACTH gel treatment as long as insurance coverage continues.

\section{Discussion}

This retrospective case review of the responses of five patients to ACTH gel in the short-term treatment of worsening dermatomyositis or polymyositis found clinically significant improvement in all patients. Effective and tolerable treatment options for patients with refractory dermatomyositis and polymyositis are limited. ACTH gel may provide a novel anti-inflammatory and immunomodulatory option, given its possible mechanisms of action beyond steroidogenesis. ${ }^{8}$

Although ACTH gel is approved for use in dermatomyositis and polymyositis, it is not well known as a potential treatment option because of a paucity of clinical data. In these five patients seen in our clinic over the past four years, the decision to use ACTH gel was motivated by increasing weakness in all patients (one patient also had worsening rash), increasing diplopia in one patient, and inadequate response to previous immunosuppressive therapies in all patients. Improvement on manual muscle testing and patientreported function in daily activities was seen in all patients, and patients uniformly reported that these changes had a significant impact on their quality of life and their level of independent function. Manual muscle testing scores have been associated with functional abilities, such as walking and completion of activities of daily living in patients with polymyositis or dermatomyositis, as well as in patients with other neuromuscular diseases. ${ }^{15-17}$ In functional terms, mean manual muscle testing strength grade $<3$ has been associated with maximal or total assistance in children with Duchenne muscular dystrophy, whereas mean muscle strength grade $\geq 3$ has been associated with relative independence in activities of daily living. ${ }^{16}$ In this retrospective clinical case series of patients with dermatomyositis or polymyositis, a score $\leq 3$ in proximal muscle groups was associated with a patient being unable to walk or complete activities of daily living independently. A score $\geq 4$ in proximal muscle groups was associated with a patient being able to ambulate independently and complete activities of daily living. Three patients who had impaired ambulation prior to ACTH gel therapy returned to independent ambulation during treatment. Two patients who were unable to care independently for a child or grandchild prior to ACTH gel therapy regained this independence. One patient regained independence in her activities of daily living, and another patient was able to return to work following ACTH gel treatment. The patient with polymyositis and extraocular muscle involvement was atypical. However, other potential diseases, including myasthenia gravis, thyroid disorders, and oculopharyngeal muscular dystrophy, were excluded. The patient showed improved proximal limb muscle strength after ACTH gel treatment for 12 weeks but no improvement in her diplopia.

These patients all showed significant improvement in short-term responses to ACTH gel. Therapy was limited to a few months in most patients, so long-term responses and side effects were not evaluated. This small, retrospective case review included patients seen during the course of patient care, and as such, limitations include open-label treatment and unblinded outcome evaluation. Because ACTH gel treatment was short-term, patients received concomitant therapies during treatment with ACTH gel and continued those therapies as maintenance treatment following cessation of ACTH gel. The short-term treatment of symptom exacerbation with ACTH gel avoided the need to increase existing immunomodulatory therapy in all cases. Though effects of concomitant medications during ACTH gel therapy cannot be ruled out, patients were on stable dosing for at least 60 days prior to initiation of ACTH gel.

All patients tolerated ACTH gel $80 \mathrm{U}$ twice weekly (once weekly for patient 5) for up to 3 months with no significant side effects and no need to taper therapy, indicating this treatment regimen may be more tolerable than high-dose corticosteroids for the short-term treatment of disease exacerbations. Patient 2 recently began a second ongoing period of ACTH gel treatment, with no side effects or change in $\mathrm{HbA}_{1 \mathrm{c}}$ over 7 months. The effectiveness of ACTH gel in patients refractory to corticosteroid therapy and in those unable to tolerate the side effects of high-dose corticosteroids suggests nonsteroidal mechanisms of action. The two patients on maintenance doses of prednisone while receiving ACTH 
gel indicate that nonsteroidal aspects of ACTH activity may be important for a clinical response in such patients. The encouraging results from this small retrospective case series suggest that ACTH gel should be considered as a therapeutic option for the treatment of dermatomyositis and polymyositis, particularly in refractory cases. A more formal, long-term examination of patient responses and side effects to treatment with ACTH gel is warranted. Additionally, given the patient-reported significance of the improved functional abilities that occurred with ACTH gel treatment, inclusion of a quality of life measure would be beneficial.

\section{Disclosure}

Lynanne McGuire of MedVal Scientific Information Services LLC provided medical writing and editorial assistance with this work.

\section{References}

1. Dalakas MC. Immunotherapy of myositis: issues, concerns and future prospects. Nat Rev Rheumatol. 2010;6(3):129-137.

2. Marie I, Mouthon L. Therapy of polymyositis and dermatomyositis. Autoimmun Rev. 2011;11(1):6-13.

3. Patwa HS, Chaudhry V, Katzberg H, Rae-Grant AD, So YT. Evidencebased guideline: intravenous immunoglobulin in the treatment of neuromuscular disorders: report of the Therapeutics and Technology Assessment Subcommittee of the American Academy of Neurology. Neurology. 2012;78(13):1009-1015.

4. Lin RY, Rodriguez-Baez G, Bhargave GA, Lin H. Intravenous gammaglobulin-associated renal impairment reported to the FDA: 2004-2009. Clin Nephrol. 2011;76(5):365-372.

5. Levine TD. Rituximab in the treatment of dermatomyositis: an open-label pilot study. Arthritis Rheum. 2005;52(2):601-607.

6. Oddis CV, Reed AM, Aggarwal R, et al. Rituximab in the treatment of refractory adult and juvenile dermatomyositis (DM) and adult polymyositis (PM) - the RIM Study [L13]. Presented at the American College of Rheumatology/Association of Rheumatology Health Professionals annual scientific meeting, November 6-10, 2010, Atlanta, GA.
7. Questcor Pharmaceuticals, Inc. HP Acthar ${ }^{\circledR}$ Gel (repository corticotropin injection, full prescribing information). Hayward, CA: Questcor Pharmaceuticals, Inc.; 2011.

8. Catania A, Lonati C, Sordi A, Carlin A, Leonardi P, Gatti S. The melanocortin system in control of inflammation. ScientificWorldJournal. 2010;10:1840-1853.

9. Baram TZ, Mitchell WG, Tournay A, Snead OC, Hanson RA, Horton EJ. High-dose corticotropin (ACTH) versus prednisone for infantile spasms: a prospective, randomized, blinded study. Pediatrics. 1996;97(3): 375-379.

10. Bomback AS, Tumlin JA, Baranski J, et al. Treatment of nephrotic syndrome with adrenocorticotropic hormone (ACTH) gel. Drug Des Devel Ther. 2011;5:147-153.

11. Simsarian JP, Saunders C, Smith DM. Five-day regimen of intramuscular or subcutaneous self-administered adrenocorticotropic hormone gel for acute exacerbations of multiple sclerosis: a prospective, randomized, open-label pilot trial. Drug Des Devel Ther. 2011;5:381-389.

12. Rider LG. Outcome assessment in the adult and juvenile idiopathic inflammatory myopathies. Rheum Dis Clin North Am. 2002;28(4): 935-977.

13. Oddis CV, Rider LG, Reed AM, et al. International consensus guidelines for trials of therapies in the idiopathic inflammatory myopathies. Arthritis Rheum. 2005;52(9):2607-2615.

14. Rider LG, Giannini EH, Brunner HI, et al. International consensus on preliminary definitions of improvement in adult and juvenile myositis. Arthritis Rheum. 2004;50(7):2281-2290.

15. Lohmann Siegel K, Hicks JE, Koziol DE, Gerber LH, Rider LG. Walking ability and its relationship to lower-extremity muscle strength in children with idiopathic inflammatory myopathies. Arch Phys Med Rehabil. 2004;85(5):767-771.

16. Uchikawa K, Liu M, Hanayama K, Tsuji T, Fujiwara T, Chino N. Functional status and muscle strength in people with Duchenne muscular dystrophy living in the community. $J$ Rehabil Med. 2004;36(3):124-129.

17. Rider LG, Koziol D, Giannini EH, et al. Validation of manual muscle testing and a subset of eight muscles for adult and juvenile idiopathic inflammatory myopathies. Arthritis Care Res (Hoboken). 2010;62(4): 465-472.

\section{Publish your work in this journal}

Drug Design, Development and Therapy is an international, peerreviewed open-access journal that spans the spectrum of drug design and development through to clinical applications. Clinical outcomes, patient safety, and programs for the development and effective, safe, and sustained use of medicines are a feature of the journal, which

\section{Dovepress}

has also been accepted for indexing on PubMed Central. The manuscript management system is completely online and includes a very quick and fair peer-review system, which is all easy to use. Visit http://www.dovepress.com/testimonials.php to read real quotes from published authors. 